Vowe, Gerhard; Wolling, Jens:

Ein Tag wie jeder andere? : die Bundestagswahl 2002 im Radio

Original erschienen in:

Holtz-Bacha, Christina (Hrsg.): Die Massenmedien im Wahlkampf : die Bundestagswahl 2002. - Wiesbaden : Westdt. Verl., 2003, S. 98-115.

ISBN (print): $3-531-14028-0$

ISBN (online): 978-3-322-80461-7

DOI: $\quad$ 10.1007/978-3-322-80461-7_6

URL: $\quad$ http://dx.doi.org/10.1007/978-3-322-80461-7_6

[Gesehen: 11.02.2016] 


\title{
Ein Tag wie jeder andere? Die Bundestagswahl 2002 im Radio
}

\author{
Gerhard Vowe \& Jens Wolling
}

\section{Einleitung: Welche publizistische Bedeutung hat das Radio?}

"Der Rundfunk ist Dauergast, und mit einem solchen macht man bekanntlich keine Umstände. Das Leben geht weiter, als wäre er gar nicht da." So charakterisierte Rudolph Arnheim das Radio bereits 1936, und da waren Servicewelle und Formatuhr noch nicht erfunden. Der Dauergast ist auch an Wahltagen anwesend. Dies ist so selbstverständlich, daß es weitgehend unbemerkt bleibt. Deshalb scheint die Frage, wie die Bundestagswahl im Radio dargestellt wird, zunächst einmal wenig relevant zu sein. In der Wahrnehmung von Öffentlichkeit und Fachöffentlichkeit ist Radio ein Unterhaltungsund Nebenbei-Medium mit niedrigem publizistischen Anspruch, geringem Informationsgehalt und vernachlässigbarer politischer Wirkung. Das Radio überhört man gerne; darauf hat es sich mehr und mehr eingerichtet. Dementsprechend dünn gestrickt ist auch die Literaturdecke im Themenfeld Wahlen und Hörfunk (vgl. Meyer \& Port, 1994), so daß dieses Gebiet zu einem weißen Fleck auf der Landkarte der kommunikationswissenschaftlichen Wahlforschung geworden ist (Holtz-Bacha, 1996, S. 34). Während Zeitungen (vgl. Schönbach, 1983) und Plakate (vgl. Lessinger, Moke \& HoltzBacha in diesem Band) zumindest als "unterschätzte Medien" anerkannt wurden, ist für das Radio eine solche Ehrenrettung noch nicht einmal versucht worden. Das war nicht immer so: So lange es noch ein neues Medium war, wurde ihm gebührende Aufmerksamkeit zuteil (vgl. z. B. Cantril, 1947; Lazarsfeld, Berelson \& Gaudet, 1944; Merton, 1946; Berelson, Lazarsfeld \& McPhee, 1954). Doch mit dem Aufkommen des Fernsehens verschwand das Radio vom Radarschirm der Forschung zur politischen Kommunikation - angesichts der ungebrochen hohen Publikumsattraktivität des Radios mit seinen großen Reichweiten und Nutzungszeiten eine fragwürdige Entwicklung. Einmal noch wurde der politischen Funktion des Radios etwas mehr Beachtung geschenkt: in den 80er Jahren, als die Öffnung des Radiomarktes den Marktzutritt kommerzieller regionaler und lokaler Sender zur Folge hatte. Ein Großteil der vorliegenden Literatur zu Politik und Radio beschäftigt sich mit eben dieser Strukturfrage (vgl. Widlok, 1994): Unter welchen strukturellen Vorgaben soll lokaler und regionaler Hörfunk möglich sein und welche Folgen wird dieses für die örtliche publizistische Vielfalt haben? 
Einige wenige Studien beleuchten den Zusammenhang von Radio und Politik unter dem Kommunikatorenaspekt (vgl. Jarren \& Donges, 1996). Zumeist geht es dabei um die Frage, in welchem Maße kommerzielle Hörfunksender auch in Zeiten verschärften Kostendrucks in der Lage sind, publizistisch relevante Leistungen zu erbringen. Auch Inhaltsanalysen von Hörfunkprogrammen sind nicht zuletzt wegen der erheblichen Dokumentationsprobleme sehr viel seltener als für andere Medienbereiche (vgl. Klingler \& Schröter, 1993) und in Deutschland stark von den Programmkontrollaufgaben der Landesmedienanstalten geprägt (vgl. Hesse, 1994, S. 150). Aus diesen Inhaltsanalysen ergibt sich folgendes Bild vom Hörfunkangebot: Bis auf die wenigen neu eingerichteten speziellen Informationswellen (z. B. "MDR-Info") sind die Programme geprägt durch die Dominanz von Musik. Dies gilt auch für den öffentlich-rechtlichen Hörfunk; allerdings ist im privaten Rundfunk der Anteil politischer Information noch stärker auf einen "Informationskernbestand" in den Nachrichten zurückgenommen (Marcinkowski, 1998, 174). Mittlerweile haben sich auch weitgehend "politikfreie" Wellen als regional oder national verbreitete Spartenprogramme etabliert. Der Vergleich zwischen öffentlich-rechtlichen und privaten Angeboten steht nach wie vor im Vordergrund der Radioinhaltsforschung und hat im Hinblick auf Themen-, Akteurs- und Meinungsvielfalt des politischen Programmanteils ein differenziertes Bild ergeben (vgl. Trebbe \& Maurer, 1999; Weiß, 1988). Darüber sind andere politisch relevante Unterscheidungen vernachlässigt worden, wie z. B. die unterschiedliche Altersgruppenorientierung von Sendern oder die Unterscheidung zwischen redaktionellen Inhalten und politischer Werbung (vgl. Holtz-Bacha, 1997; Jarren \& Bode, 1996, S. 74 ff.; Müller, 1999, S. 257). Über die Programmstruktur speziell in Wahlzeiten wissen wir wenig: Offenbar dominiert auch dann die Musik (Lohmann, 1987), aber in welchem Maße z. B. ein Regierungsbonus zu verzeichnen ist, ob Journalisten oder Politiker mehr zu Wort kommen, ob die Kandidaten oder die Wahlkampfthemen stärker im Vordergrund stehen, ob einzelne Themen konsonant die Nachrichten beherrschen oder jeder Sender seine eigene Themenagenda herausbildet - alles dies wissen wir vom Radio nicht und können hier keinen Vergleich mit anderen Medien ziehen.

In aktuellen politischen Wirkungsstudien fehlt das Radio zumeist, auch wenn sie multimedial angelegt sind. Wahlkampfstudien thematisieren im Regelfalle Fernsehen und Tagespresse, vielleicht auch noch das Internet und Plakate, nicht aber den Hörfunk (vgl. z. B. Holtz-Bacha, 1999; Rettich \& Schatz, 1998; Dahlem, 2001; Denton, 1998). Wenn der Hörfunk doch einmal berücksichtigt wird, so verschwindet die feststellbare Wirkung zumeist hinter der von Fernsehen und Presse (z. B. Ohr \& Schrott, 2001; Scherer, 1995). Es konnten aber auch eigenständige Wirkungen des Hörfunks nachgewiesen werden. So beeinflußt bei Semetko und Schönbach (1994, S. 111) die Zuwendung zum Radio die Sympathiewerte für Parteien, wenn auch in geringerem Maße als andere Medien. In einer anderen Studie fällt der kognitive Effekt im Vergleich zur Presse schwächer aus, im Vergleich zum Fernsehen aber stärker (Becker \& Dunwoody, 1982, S. 215). Die gleichen Relationen findet Schulz (1998, S. 533) im Hinblick auf das politische Interesse und die Einflußüberzeugung. Wirkungsstudien, die sich allein mit dem Hörfunk befassen, sind sehr selten, sie beschränken sich zudem häufig auf die Erinnerung 
von Hörfunknachrichten (vgl. Schönbach \& Goertz, 1995; Kindel, 1998) oder auf sehr spezielle Aspekte, wie die Wirkung des Talk-Radio auf politische Einstellungen (Hollander, 1996) oder den unterschiedlichen Einfluß positiver und negativer politischer Werbespots (Shapiro \& Rieger, 1992). Die deutliche Distanz der Wirkungsforschung zum Radio liegt auch daran, daß die vermuteten politischen Wirkungen des Radios eher subtil sind. Es wird keine kurzfristige Veränderung von Wissen, Einstellungen oder Aktivitäten erwartet, sondern z. B. die "Synchronisierung" der Bürger auch unter politischem Aspekt: Gerade das Radio mit seinem stetigen Informationsstrom trage dazu bei, $\mathrm{da}$ dem politischen Wissen und Meinen in einer Gesellschaft ein gemeinsames Zeitgerüst unterliege (Hasebrink, 1994, S. 170).

Über politische relevante Aspekte der Radionutzung wissen wir hingegen vergleichsweise viel (vgl. Hasebrink, 1994; Gleich, 1995; Gleich, 2000). Dies ist vor allem der "Langzeitstudie Massenkommunikation" zu verdanken, die neben Tageszeitung und Fernsehen immer auch den Hörfunk im Blick behielt (Kiefer, 1996; Ridder \& Engel, 2001). Aber auch Marktforschungsdaten (z. B. aus der Media Analyse) zu der Verteilung der Hörerschaft auf Sender, Zeitpunkte und Nutzungssituationen sind im Hinblick auf politisch-publizistische Belange relevant. Vor allem die Motive für die Zuwendung zum Radio sind gut untersucht. Generell läßt sich sagen, daß die meisten Rezipienten von einem Radioprogramm erwarten, daß es neben anderen Tätigkeiten genutzt werden kann und daß die Musik nach ihrem Geschmack ist. Die Musikmischung ist das Kriterium, nach dem entschieden wird, ob man einem Sender treu bleibt oder ihn wechselt. Vor allem dadurch ergibt sich eine starke Fragmentierung des Hörfunkpublikums nach Alters- und Motivgruppen (vgl. Oehmichen, 1999) und dementsprechend eine Differenzierung des Angebots (vgl. Klingler \& Windgasse, 1994, S. 105). Doch die Erwartungen der Hörer an das Radio gehen über eine bestimmte Musikmischung hinaus. Schon an zweiter Stelle der Wichtigkeit folgen Informationsleistungen; vor allem aktuelle Informationen in Form von regelmäßigen Nachrichtensendungen werden vom Radio erwartet. Radio wird komplementär zu anderen Informationsmedien genutzt, und zwar vor allem für die thematisch nicht spezifizierte Grundinformation am frühen Morgen und für die laufende schnelle Aktualisierung (vgl. Klingler \& Windgasse, 1994, S. 121). Damit erfüllt der Hörfunk vor allem das Bedürfnis, den sozialen Erwartungen zu entsprechen, aktuell informiert zu sein (Ohr \& Schrott, 2001). Es wird also von einem Sender erwartet, daß er Nachrichten bringt und dabei (stunden)aktuell bleibt, aber die Qualität der Nachrichten ist im allgemeinen kein Kriterium der Auswahl unter Sendern - dies bleibt der Musikfarbe und nachgeordnet dazu der Moderation überlassen (Ecke \& Stuiber, 1995).

Diese Motivlage schlägt sich im Nutzungsverhalten nieder: Mehr als die Hälfte der Bevölkerung nutzen täglich das aktuelle Informationsangebot des Radios. Die Zahl der Personen, die von sich behaupten, regelmäßig politische Informationen im Radio zu hören, ist größer als die Zahl derjenigen, die angeben, regelmäßig Musik im Radio zu hören (Kiefer, 1996, S. 207). Das Radio erreicht mit seinen in großflächige Magazinsendungen eingestreuten Informationsleistungen gerade auch die eher unterhaltungsorien- 
tierten Teile des Publikums (Berens, Kiefer \& Meder, 1997) und diejenigen, die nicht aktiv nach politischer Information suchen. Die Radionutzung korreliert im Vergleich zur Zeitungsnutzung nur schwach mit dem politischen Interesse (vgl. Schulz, 2001, S. 179). Die unter 20jährigen werden noch am ehesten von den Informationsangeboten des Hörfunks erreicht (Holtz-Bacha, 1997, S. 298). Gerade also mit Blick auf diejenigen, die Zeitungen und politische Informationsangebote im Fernsehen meiden, ist dem Radio eine wichtige Aufgabe erwachsen. Von daher ist das Radio zumindest aus der Sicht des Publikums ein Medium mit publizistischer Relevanz - wenn auch mit deutlichem Abstand in Bedeutung, Kompetenz und Glaubwürdigkeit zu Fernsehen und Tageszeitung. Auch in Wahlzeiten wird es als Informationsquelle von den Rezipienten dem Fernsehen und der Tageszeitung nachgeordnet (Schulz, Zeh \& Quiring, 2000, S. 417 f., S. 422). Das ändert jedoch nicht daran, daß erst die Kombination der aktuellen Medien die breite, kontinuierliche politische Information der Bevölkerung sichert.

Doch in welchem Maße sind die Sender in der Lage und willens, diesen Informationsbedarf zu decken? Diese Frage stellt sich besonders dringlich im Zusammenhang mit politischen Wahlen, den Hochzeiten des demokratischen politischen Prozesses.

\section{Methode: Verschränkung von quantitativer und qualitativer Inhaltsanalyse}

Um diese Frage zu beantworten, werden wir im folgenden wahlspezifische Teilergebnisse einer quantitativen Inhaltsanalyse darstellen und durch eine qualitative Inhaltsanalyse ergänzen. Die Untersuchung entstand im Rahmen eines Forschungsprojekts, in dem die Erwartungen der Hörer an das Radio und die Leistungen der Anbieter unter Qualitätsaspekten untersucht werden. Dieses Projekt wird von der Thüringer Landesmedienanstalt im Verbund mit den Landesmedienanstalten in Hessen und SachsenAnhalt finanziert. Da bei dieser Untersuchung die Analyse der Qualität von Radioprogrammen im Mittelpunkt steht, ist die nachfolgend präsentierte Auswertung der Wahlthematisierung eine Sekundäranalyse der Daten, denn die Studie ist nicht speziell für diesen Zweck konzipiert worden.

Erhoben und ausgewertet wurden die Daten von sechs Radiosendern, die ein Programm (auch) für Thüringen ausstrahlen. ${ }^{1}$ Dabei handelt es sich um folgende Sender:

- Antenne Tbüringen (AT) ist der reichenweitenstärkste Sender in Thüringen (30,9\% Reichweite nach MA 2002/II). AT hat 1993 den Sendebetrieb aufgenommen und bietet für die Zielgruppe im Alter von 14-49 Jahren ein Programm mit einem Verhältnis von Wort- zu Musikanteil von 30/70 und einem Musikformat "HOT-AC" (Adult Contemporary).

1 Aus organisatorischen Gründen ist es nicht möglich gewesen, die Programme der Sender aus SachsenAnhalt und Hessen in die Analyse einzubeziehen. Nicht analysiert wurde außerdem die Informationswelle "MDR-Info". 
- Der zweite - mit 12,4 Prozent Reichweite aber deutlich kleinere - privatwirtschaftliche Sender ist die Landeswelle Thüringen (LW). Die LW ging 1995 auf Sendung und bietet für die Zielgruppe im Alter von 20-49 Jahren ein Programm mit einem Verhältnis von Wort- zu Musikanteil von 30/70 und einem "Soft-AC-Oldie based"Musikformat.

- Der dritte privatwirtschaftlich organisierte Sender ist radio TOP 40 (T40). Der Sender wird im gleichen Haus wie Antenne Thüringen produziert und kann als deren Jugendwelle bezeichnet werden. Die Zielgruppe des Senders sind die 14- bis 25jährigen, die er mit einem Verhältnis von Wort- zu Musikanteil von 20/80 und einer "Alternativ-Mainstream"-Musikfarbe (Techno, Hip Hop, Alternative Rock etc.) zu ereichen sucht.

- Auch der öffentlich-rechtliche Rundfunk (MDR) ist in der Untersuchung mit drei Sendern vertreten. Der reichweitenstärkste Sender des MDR in Thüringen $(21,2 \%)$ ist das Landesprogramm MDR 1 Radio Tbüringen (MDR1). Es handelt sich um ein Programm für die Altersgruppe ab 30 Jahre mit einer Wort/Musik-Mischung von $30 / 70$ und ist geprägt durch populäre Schlagermusik und deutschen Schlager.

- An zweiter Stelle - fast gleichauf mit MDR1 - folgt JUMP (19,8\%), ein Programm, das gemeinsam für die drei Bundesländer Sachsen, Sachsen-Anhalt und Thüringen produziert wird und mit einem "Young HOT- AC"-Musikformat die Zielgruppe der 20- bis 39jährigen ansprechen möchte. Das Verhältnis von Wort zu Musik beträgt hier knapp 30/70.

- Das dritte untersuchte öffentlich-rechtliche Programm ist MDR Kultur (MK) ebenfalls für die drei Länder des MDR produziert. Das werbefreie Programm sendet seit 1992, ist in der Musikfarbe klassikorientiert und möchte mit einer Wort/Musik-Relation von ca. 40/60 die Zielgruppe der ab 30jährigen erreichen.

Analysiert wurde von allen sechs Sendern das Programm an zwei Tagen vor der Wahl, am Wahltag und an einem Tag nach der Wahl. Codiert wurden jeweils 14 Stunden von 5 bis 19 Uhr. Am Wahltag wurde die Analyse auf 19 Stunden - bis 24 Uhr - ausgedehnt.

Gegenstand der nachfolgenden Analysen ist die gesamte Berichterstattung mit Wablbezug. Ein Wahlbezug wurde nur dann als gegeben angesehen, wenn durch die Erwähnung der Bundestagswahl explizit ein solcher Bezug hergestellt wurde. Wenn beispielsweise in einem Beitrag nur von einer Initiative des Kanzlerkandidaten oder von einer Stellungnahme des Kanzlers die Rede war, diese Äußerungen aber weder von den Politikern, noch von den Journalisten mit der Bundestagswahl in Verbindung gebracht wurden, dann wurde dies nicht als Wahlbeitrag codiert. Zusätzlich wurden in der Analyse jene Beiträge aus der Nachwahlzeit berücksichtigt, bei denen es um die Koalitionsverhandlungen zwischen den Grünen und der SPD ging, unabhängig davon, ob dabei nochmals die Wahl erwähnt wurde oder nicht. Diese Definition ist eine relativ enge 
Festlegung dessen, was unter "Wahlbezug" zu verstehen ist (vgl. dazu die sehr ähnliche Definition bei Krüger \& Schramm, 2002, S. 610). Wenn man die Definition von "Wahlbezug" jedoch weiter faßt, ist man sehr schnell in der Situation, daß so ziemlich alles, was sich in der Vorwahlzeit ereignet und auch nur im Entferntesten mit Politik in Deutschland zu tun hat, als "Wahlkampf" codiert werden müßte. Durch eine solche Ausweitung des Definitionsbereichs könnte aber kaum noch ermittelt werden, was über die normale Politikberichterstattung hinausgeht - was also das Besondere in Wahlzeiten ist. Der Kontrast zur normalen Berichterstattung würde verwischt. Diese enge Definition ist bei der Interpretation der Ergebnisse zu berücksichtigen. Wenig Berichterstattung über die Wahl bedeutet nicht, daß nicht über Politik berichtet wurde, sondern nur, daß die Wahl in diesem Zusammenhang keine Rolle gespielt hat.

Inhaltlich wurde der Analysebereich also eng gefaßt; vom Genre her wurde er hingegen weit gefaßt: Wir haben Informationsbeiträge, humorvolle Beiträge, Hörermeinungen zur Bundestagswahl, Trailer, mit denen Wahlbeiträge oder Wahlsondersendungen angekündigt wurden, und Wahlwerbung berücksichtigt. In die Kategorie Informationsbeiträge wurden sowohl Nachrichtenbeiträge eingeordnet als auch einzelne Beiträge, die außerhalb des Nachrichtenblocks gesendet wurden.

Die Analyse der Wahlthematisierung stand vor einem zentralen methodischen Problem. Aufgrund der wenigen Untersuchungstage sind einer quantitativen Inhaltsanalyse, deren Ziel es ist, verallgemeinerungsfähige Ergebnisse zu erzielen, enge Grenzen gesetzt. Nach den ersten Auszählungen zeigte sich zwar, daß durchaus eine beachtliche Anzahl von Beiträgen ermittelt und codiert worden war (Tabelle 1). Es stellte sich jedoch heraus, daß es sich bei einem Großteil dieser Beiträge um Wiederholungen von ähnlichen oder sogar identischen Beiträgen handelte. Dies ist vor allem bei den Meldungen im Rahmen von Nachrichtensendungen der Fall, aber auch bei den an anderer Stelle ins Programm eingestreuten Beiträgen. Die Zahl der ermittelten Originalbeiträge war bei allen Sendern so niedrig, daß jede weitergehende quantitative Auswertung mit sehr kleinen Fallzahlen hätte vorlieb nehmen müssen oder man ähnliche oder identische Beiträge mehrfach in der Analyse hätte berücksichtigen müssen. Auf der Basis der vorhandenen Daten konnten somit verallgemeinerungsfähige Ergebnisse nicht erzielt werden. Eine weitergehende quantitative Auswertung, z. B. hinsichtlich der politischen Akteure, des thematischen Zusammenhangs der Beiträge, des Anteils an Hintergrundinformation oder der Konflikthaltigkeit war somit nicht sinnvoll.

$\mathrm{Da}$ jedoch dem Radio in der Wahlforschung bislang kaum Aufmerksamkeit geschenkt worden ist, erschien es lohnend, die Analysen hier nicht abzubrechen, sondern die ermittelten Beiträge intensiver und genauer zu analysieren. Auf diese Weise sollte geprüft werden, inwieweit die gängigen Kategorien der Inhaltsanalyse (vor allem Themen, Akteure, Wertungen, Nachrichtenfaktoren) ausreichen, wesentliche Merkmale der Wahlthematisierung im Radio zu beschreiben, oder ob weitere Kategorien notwendig sind, um die Eigenarten der Wahlthematisierung im Radio angemessen zu erfassen. Wie noch zu zeigen sein wird, rechtfertigen die dabei ermittelten Ergebnisse diese Vorgehensweise: Zum einen wird durch den genaueren Blick deutlich, auf wie vielfältige 
Weise die Wahl im Radio thematisiert wurde. Trotz der wenigen Untersuchungstage entsteht ein differenziertes Bild der Wahlthematisierung im Radio. Auf der anderen Seite verdeutlicht der häufig sehr eigenwillige Charakter der analysierten Beiträge, daß bei Inhaltsanalysen im Radio zusätzliche Kategorien berücksichtigt werden sollten.

\section{Ergebnisse}

\subsection{Gesamtumfang der Wablthematisierung}

In der nachfolgenden Tabelle werden die Anzahl und der zeitliche Umfang der Beiträge mit Wahlbezug in den sechs Thüringer Sendern an den vier Untersuchungstagen dargestellt.

Tabelle 1: Der Umfang der Wahlthematisierung

\begin{tabular}{|c|c|c|c|c|c|c|c|c|c|c|c|c|c|}
\hline & \multicolumn{2}{|c|}{$\begin{array}{l}\text { Landes- } \\
\text { welle } \\
\text { Thüringen }\end{array}$} & \multicolumn{2}{|c|}{$\begin{array}{l}\text { Antenne } \\
\text { Thüringen }\end{array}$} & \multicolumn{2}{|c|}{ TOP 40} & \multicolumn{2}{|c|}{ JUMP } & \multicolumn{2}{|c|}{$\begin{array}{c}\text { MDR 1 } \\
\text { Radio Thü- } \\
\text { ringen }\end{array}$} & \multicolumn{2}{|c|}{$\begin{array}{l}\text { MDR } \\
\text { Kultur }\end{array}$} \\
\hline & & $\mathrm{N}$ & $\mathrm{D}$ & $\mathrm{N}$ & D) & $\mathrm{N}$ & $\mathrm{D}$ & $\mathrm{N}$ & $\mathrm{D}$ & $\mathrm{N}$ & $\mathrm{D}$ & $\mathrm{N}$ & $\mathrm{D}$ \\
\hline \multirow{6}{*}{16.9.} & Infobeiträge & $37(4)$ & 25 & $17(3)$ & 13 & 11(2) & 4 & $18(2)$ & 8 & $16(4)$ & 18 & $16(1)$ & 14 \\
\hline & Hörerbeteiligung & 5 & 3 & & & & & & & & & & \\
\hline & Trailer & 16 & 7 & & & & & & & 2 & 1 & & \\
\hline & Humor & 5 & 5 & 4 & 7 & & & 8 & 6 & 1 & 2 & 2 & 7 \\
\hline & Wahlwerbung & & & 8 & 6 & & & 2 & 4 & & & & \\
\hline & Summe & 61 & 40 & 29 & 26 & 11 & 4 & 28 & 18 & 19. & 21 & 18. & 21. \\
\hline \multirow{6}{*}{19.9.} & Infobeiträge & $13(5)$ & 7 & $5(2)$ & 4 & & & $3(2)$ & 4 & $13(4)$ & 12 & $5(4)$ & 11 \\
\hline & Hörerbeteiligung & 5 & 3 & 4 & 6 & & & & & & & & \\
\hline & Trailer & 2 & 1 & 1 & 1 & & & & & & & & \\
\hline & Humor & 1 & 2 & 6 & 8 & & & & & 1 & 1 & & \\
\hline & Wahlwerbung & & & 10 & 7 & & & & & & & & \\
\hline & Summe & 21 & 13 & 26 & 26 & 0 & 0 & 3 & 4 & 13 & 12. & 5 & 11 \\
\hline \multirow{4}{*}{$\begin{array}{l}22.9 . \\
5-18 \\
\text { Uhr }\end{array}$} & Infobeiträge & $40(11)$ & 36 & $19(7)$ & 20 & & & $32(5)$ & 15 & $21(9)$ & 27 & $11(3)$ & 15 \\
\hline & Trailer & 5 & 2 & & & & & & & & & & \\
\hline & Humor & 2 & 4 & & & & & 1 & 1 & & & & \\
\hline & Summe & 47 & 42 & 19 & 20 & 0 & 0 & 33 & 16 & 21 & 27 & 11 & 15 \\
\hline \multirow{3}{*}{$\begin{array}{l}22.9 \\
18 . \\
24 \\
\text { Uhr }\end{array}$} & Infobeiträge ${ }^{2}$ & 16 & 32 & 25 & 37 & & & 29 & 35 & 71 & 119 & 2 & 3 \\
\hline & Trailer & 3 & 1 & & & & & & & & & & \\
\hline & Summe & 19 & 33 & 25 & 37 & 0 & 0 & 29 & 35 & 71 & 119 & 2 & 3 \\
\hline \multirow{2}{*}{25.9.} & Infobeiträge & $28(1)$ & 16 & $9(1)$ & 7 & $6(1)$ & 2 & $20(3)$ & 6 & $31(6)$ & 24 & $20(7)$ & 28 \\
\hline & Summe & 28 & 16 & 9 & 7 & 6 & 2 & 20 & 6 & 31 & 24 & 20 & 28 \\
\hline \multicolumn{2}{|c|}{ Gesumbumme } & 176 & & 108 & 25 & 17 & 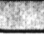 & 113 & $n$ & 155 & 3 & 56 & $a s$ \\
\hline
\end{tabular}

$N=$ Anzahl der Beiträge (In Klammern: Anzahl der Originalbeiträge ohne ähnliche und identische Wiederholungen)

$\mathrm{D}=$ Gesamtdauer der Beiträge in Minuten

2 Am Wahlabend war die klare Abgrenzung von Originalbeiträgen schwierig, da häufig einzelne 'l'eile der Beiträge neue Themenaspekte behandelten, andere Flemente aber wiederholt wurden. Insgesamt ging es aber bei allen Beiträgen um den Wahlausgang. 


\subsection{Vorvablzeit}

Aus der unmittelbaren Vorwahlzeit wurden Montag, der 16.9., und Donnerstag, der 19.9., analysiert. Die beiden Tage unterscheiden sich deutlich in ihrer Themenstruktur, wenn man die Beiträge grob in folgende Kategorien einteilt:

- Themen des Wahlkampfes: Hierunter verstehen wir Beiträge, die auf ein bestimmtes politisch-substantielles Thema orientiert sind, z. B. Zuwanderung.

- Wahlkampf als Thema: Hierunter werden Beiträge gefaßt, die den Wahlkampf selbst zum Thema nehmen, z. B. die Wahlchancen einer Partei.

- Skurrile Themen: Hierunter werden Beiträge gefaßt, die eher skurrile Aspekte der Wahl bzw. des Wahlkampfes zum Thema haben oder die ein normales Wahlthema in einer eher skurrilen Form behandeln.

- Humorige Beiträge: Hierunter werden Beiträge gefaßt, in denen die Wahl zum Anlaß genommen wird, um die Hörer zum Lachen oder zumindest zum Schmunzeln zu bringen.

Der zentrale Unterschied zwischen den beiden Tagen bestand darin, daß der erste Tag von einem einzelnen Thema dominiert wurde, der zweite nicht. Dadurch lag die Anzahl der Beiträge mit Wahlbezug am zweiten Untersuchungstag bei allen Sendern niedriger. Zwar war die Anzahl der Originalbeiträge nahezu unverändert, aber sie wurden nicht so häufig wiederholt wie am ersten Tag.

\subsubsection{Themen des Wahlkampfs}

Am ersten Untersuchungstag spielte ein wahlbezogenes Thema in der Berichterstattung aller sechs untersuchter Sender eine zentrale Rolle (Tabelle 2), und zwar die Zuwanderungsinitiative der CDU/CSU. Inhaltlich ging es in der Berichterstattung um das Vorhaben der Union, im Falle eines Wahlsieges das Zuwanderungsgesetz von Rot-Grün rückgängig machen zu wollen, sowie um die Reaktionen auf diesen Vorstoß - teilweise auch in Form von O-Tönen - durch Mitglieder der Regierungsparteien. Sie warfen der Union vor, mit dem sensiblen Thema auf Stimmenfang gehen zu wollen. Über den Inhalt der Initiative wurde relativ wenig berichtet. Eine gewisse Ausnahme bildet hier MDR1, bei dem sich die Berichterstattung über das Thema im Lauf des Tages allmählich weiterentwickelte und dabei auch über inhaltliche Details berichtet wurde. Die Verbindung zwischen der Initiative der Union und der bevorstehenden Wahl wurde fast immer durch die Anmoderation ("Sechs Tage vor der Wahl...") und durch die Kritiker aus dem Regierungslager hergestellt. Durch die Verknüpfung des Themas mit dem Wahlkampf wurde die Initiative weniger als inhaltlich und mehr als wahltaktisch motiviert dargestellt.

Das zweite große Thema mit Wahlbezug, das am ersten Tag eine Rolle spielte, war die Ankündigung der Bundesregierung, dass durch die Übernahme von Staatsbürg- 
schaften zur Rettung der Firma Mobilcom beigetragen werden soll. Darüber wurde von allen sechs Sendern berichtet, allerdings haben dabei nur zwei das Thema explizit in den Zusammenhang mit der Wahl gebracht (AT und T40). Bei den anderen vier Sendern wurde in den Beiträgen über Mobilcom keine Beziehung zur Wahl hergestellt; sicherlich auch deshalb, weil die Opposition die Regierung zwar kritisierte, aber in keinem der ausgestrahlten O-Töne der Vorwurf erhoben wurde, die Regierung würde aus wahltaktischen Gründen Mobilcom stützen.

Tabelle 2: Die Zuwanderungsinitiative der CDU/CSU und die Mobilcom-Rettung: Anzahl der Informationsbeiträge am 16.9.

\begin{tabular}{|l|c|c|c|c|c|c|}
\hline & $\begin{array}{c}\text { Landes- } \\
\text { welle } \\
\text { Thüringen }\end{array}$ & $\begin{array}{c}\text { Antenne } \\
\text { Thüringen }\end{array}$ & TOP 40 & JUMP & $\begin{array}{c}\text { MDR 1 } \\
\text { Radio } \\
\text { Thüringen }\end{array}$ & $\begin{array}{c}\text { MDR } \\
\text { Kultur }\end{array}$ \\
\hline $\begin{array}{l}\text { Beiträge zur CDU- Zuwan- } \\
\text { derungsinitiative }\end{array}$ & 24 & 10 & 4 & 17 & 12 & 16 \\
\hline Mobilcom mit Wahlbezug & - & 6 & 7 & - & - & - \\
\hline Mobilcom obne Wahlbezug & 26 & 15 & - & 27 & 21 & 14 \\
\hline $\begin{array}{l}\text { Sonstige Beiträge mit Wahl- } \\
\text { bezug }\end{array}$ & 13 & 1 & - & 1 & 4 & - \\
\hline
\end{tabular}

Am z2veiten Tag hingegen waren Beiträge, in denen politische Themen mit der Wahl in Verbindung gebracht wurden, äußerst selten; zudem waren sie uneinheitlich verteilt. Bei der LW waren drei thematische Beiträge zu finden, in denen von den Journalisten oder von anderen Akteuren eine Verbindung mit der Wahl hergestellt wurde, z. B. die geänderte Haltung Stoibers zum bevorstehenden Irak-Krieg oder die Kritik des Zentralrats der Juden an der FDP-Führung, die sich nicht deutlich genug vom "Stimmenfang Möllemanns in rechten Gewässern" distanziert habe.

\subsubsection{Wahlkampf als Thema}

Soweit am ersten Tag überhaupt weitere Beiträge zur Wahl gesendet wurden, drehten sich diese im wesentlichen um Wahl und Wahlkampf selbst. Bei AT ging es z. B. um die Belastung der Kandidaten durch Wahlkampfauftritte oder um eine Wahlprognose, nach der die SPD Stimmen hinzugewonnen habe. Bei JUMP wurde darüber berichtet, daß die FDP angesichts aktueller Umfragewerte Abstand von ihrem Wahlziel "18 Prozent" genommen habe. Das Scheitern des ehrgeizigen Ziels wurde vom JUMP-Moderator auf eine fehlende klare Wahlaussage zurückgeführt.

3 Diese Beiträge sind nicht 'l'eil der Wahlthematisierung, weil in diesen Beiträgen kein Wahlbezug hergestellt wurde. Die Beiträge wurden hier nur aufgeführt, um zu verdeutlichen, daß das Thema bei allen Sendern eine Rolle spielte, aber eben nicht durchgängig als Wahlthema. 
Am zpeiten Tag finden sich in dieser Klasse vor allem Beiträge, in denen über Verfahrensfragen berichtet wurde. So haben AT und JUMP in jeweils einem Beitrag Informationen zum Ablauf und zur wachsenden Bedeutung der Briefwahl vermittelt. Zum anderen wurde auch direkt über den Wahlkampf informiert: MDR1 berichtete beispielsweise über die Wahlkampfabschlußkundgebung der PDS und der Grünen in Thüringen. Diese beiden Beiträge (zweimal bzw. siebenmal gesendet), sind insofern hervorzuheben, weil diese die einzigen im Untersuchungszeitraum waren, in denen programmatische Wahlkampfaussagen der Parteien unmittelbar kommuniziert wurden. Eine besondere Facette der Thematisierung von Wahlkampf steuerte MK bei: Drei in Deutschland lebende Ausländer verglichen am Beispiel von Fernsehwahlwerbung die politische Kultur in Deutschland mit der in ihren Heimatländern.

\subsubsection{Skurrile Themen}

Nur bei der LW war am 16.9. neben der Berichterstattung über die beiden genannten Wahlkampfthemen noch ein weiteres Schwerpunktthema zu finden. Dies steht hier für eine Klasse von Beiträgen, die sich um eher skurrile, randständige Themen ranken. In diesem Falle ging es um den Kauf und Verkauf von Wählerstimmen. Dieser Beitrag wurde über mehrere Stunden angekündigt und bestand vor allem aus einem kurzen Telefoninterview mit einem Mann, der angeblich seine Stimme per Internet zum Verkauf angeboten hatte. Der Moderator bezeichnete ein solches Verhalten als skandalös. Darüber hinaus wurden auch Hörermeinungen zu dem "Problem" eingeholt. Diese Hörermeinungen wurden dann über den ganzen Tag im Rahmen der dazugehörigen Beiträge immer wieder eingespielt und am Nachmittag dann auch noch für die Ankündigung der nächsten Morgenshow verwendet. Ein weiteres Beispiel für diesen Beitragstyp ist ein Telefoninterview mit dem thüringischen Justizminister, der erläuterte, wie für die Insassen der Gefängnisse die Wahl organisiert wird.

Eine andere Spielart dieser Gruppe ist das Aufgreifen von Themen in einer eher skurrilen Form. Als Beispiel kann ein Beitrag ebenfalls von der LW dienen, in dessen Mittelpunkt ein "Interview" stand, das ein Kind mit dem Erfurter Bundestagsabgeordneten Carsten Schneider (SPD) führte. Er wurde u. a. nach seinem Auto, seiner Freundin, seinem Einkommen und den Motiven für seine Kandidatur gefragt. Nicht von ungefähr stammen alle Beispiele von der LW: An ihrer Wahlthematisierung fällt auf, $\mathrm{da} ß$ häufig weder eine klare inhaltliche Abgrenzung der Beiträge, noch eine formale Unterscheidung der Beitragstypen möglich war: Der Übergang zwischen Informationsbeitrag, Hörerbeteiligung und Trailer war fließend.

Am 19.9. war es vor allem die Wahlkampfberichterstattung von MK, die sich als sehr eigenwillig herausstellte. So erhielten einige Schriftsteller die Gelegenheit, ihre Meinung zu den Spitzenkandidaten in möglichst geistreicher und witziger Art abzugeben. Auch ein Beitrag über die Wahlkampfberichterstattung der Wochenzeitschrift "Freitag" war eher in einem feuilletonistisch-ironischen als in einem nüchtern-analysierenden Stil gehalten. 


\subsubsection{Humorige Beiträge}

Bemerkenswert ist, welche Bedeutung Humor für die Thematisierung der Wahl hatte im übrigen nicht nur in der Vorwahlzeit, sondern auch am Wahltag selbst. Dabei kann die Grenze zwischen humorvollen und ernst gemeinten Beiträgen nicht immer klar gezogen werden. Einige dieser Beiträge sollen etwas genauer geschildert werden, denn nur dann wird deutlich, daß diese Spielart der Wahlthematisierung mit den gängigen Kategorien nicht erfaßt werden kann.

Bei $A T$ wurden an den zwei Tagen insgesamt sechs humorvolle Beiträge gesendet. Dreimal drehte es sich dabei um die Wahlkampfaktivitäten einer fiktiven Partei, z. B. um die Vortäuschung eines Einbruchs in die Parteizentrale dieser Partei. Dazu wurde bei einem Schlüsseldienst angerufen und nach dessen Bereitschaft gefragt, diesen durchzuführen. Auf der Grenze von Humor und Ernsthaftigkeit bewegten sich mehrere Beiträge über die Eigenschaften und Eigenarten der Kanzler- und der Kandidatengattin.

Auch bei der $L W$ spielte Humor eine wichtige Rolle. So gab es ein Telefongespräch, in dem Kanzler und Kandidat durch Stimmenimitatoren dargestellt wurden. Des weiteren wurde ein Spielformat ankündigt, bei dem die Zuhörer anrufen und zwischen Schnitzel und Weißwurst wählen sollten. In einem anderen Beitrag wurde der Ablauf im Wahllokal in etwas anzüglicher Weise dargestellt unter dem Motto: "Wie wähle ich richtig?" Hinterlegt war der Beitrag mit der Erkennungsmelodie von "Der Sendung mit der Maus". Auffällig war bei der LW, daß in den Trailern, in denen auf die eigene Wahlthematisierung hingewiesen wurde, ernsthafte und humorvolle Beiträge gleichrangig und in einem Atemzug genannt wurden.

Aber nicht nur bei den privatwirtschaftlich organisierten Sendern, auch bei den öffentlich-rechtlichen wurden humorvolle Beiträge zur Wahl gesendet. So erläuterten bei MDR 1 Kinder die Funktion von Wahlplakaten und Wahlurnen. Bei MK wurde der Beitrag eines Kabarettisten gesendet, der zur Wahlabstinenz aufrief, weil - egal wen man wählt - der Gewählte versagen werde. Auch bei JUMP wurden mehrere humorvolle Beiträge ausgestrahlt; $u$. a. wurde vermutet, daß viele Wähler in der Kabine wie beim Lotto sechs Kreuze machen würden, weil sie nicht wüßten, wen sie wählen sollen.

\subsection{Berichterstattung am Wablsonntag}

Bei Ausmaß und Qualität der Berichterstattung am Wahlsonntag zeigen sich noch stärkere Divergenzen zwischen den Radiosendern als in der Vorwahlzeit.

Bei T40 fanden die Wahlen am Wahlsonntag überhaupt nicht statt. Bei $M K$ beschränkte sich die Berichterstattung weitgehend auf grundlegende Fakten wie die Zahl der Wahlberechtigten und der Kandidaten. Später wurden dann noch Ergebnisse zur Wahlbeteiligung gesendet. Nach der Schließung der Wahllokale brachte MK nur noch eine Prognose, später die erste Hochrechnung und Stellungnahmen von den im Bun- 
destag vertretenen Parteien. Statt eigener Berichterstattung verwies MK während des ganzen Tages auf die Berichterstattung von MDR-Info. Im Vergleich dazu haben die drei großen AC-Sender und MDR1 ausführlicher über die Wahl berichtet. Die Basisinformationen zu den Wahlen wurden durch weitere Beiträge ergänzt, die der Berichterstattung jeweils einen senderspezifischen Charakter gaben.

Bei JUMP wurden die grundlegenden Informationen sehr häufig gesendet. Darüber hinausgehende Beiträge gab es jedoch nur wenige; so wurde über einen versuchten Wahlbetrug in Hamburg berichtet. Nach Schließung der Wahllokale informierte JUMP kontinuierlich über Prognosen, Hochrechnungen und erste Reaktionen der Spitzenpolitiker sowie über die Stimmung auf den Thüringer Wahlpartys. Durch Statements der Ministerpräsidenten von Sachsen-Anhalt und Sachsen und die Präsentation von Wahlergebnissen aus Ostdeutschland wurde ein regionaler Bezug hergestellt. Besondere Aufmerksamkeit schenkte JUMP auch den Reaktionen der FDP auf ihr schlechtes Abschneiden.

Etwas umfangreicher und vielfältiger war die Thematisierung der Wahl bei $A T$ : So wurden am Nachmittag O-Töne von Thüringer Bürgern zu den Motiven ihrer Wahlbeteiligung gesendet; in mehreren Beiträgen wurde die Rolle der Wahlhelfer thematisiert; dann wurde berichtet, wie Schröder und Stoiber den Wahlsonntag verbringen und wie sie die Wahlchancen ihrer Parteien einschätzen. Ein Wahlaufruf des Ministerpräsidenten Vogel wurde gesendet, der nicht nur zur Wahl aufrief, sondern auch die SPDBundesministerin Däubler-Gmelin kritisierte. Auch sonst wurden mehrfach im Laufe des Tages die Affären um Däubler-Gmelin und Möllemann in direkten Zusammenhang mit der Wahl gebracht und als belastend für SPD und FDP bezeichnet. Die überraschend politische Ausrichtung dieser Beiträge war das auffäligste Merkmal der Berichterstattung auf AT am Wahltag.

Am Abend beschränkte sich AT weitgehend auf das Standardprogramm und ergänzte dies um einige wenige Beiträge, z. B. um zwei Interviews mit dem Ministerpräsidenten und dem Vorsitzenden der Thüringer SPD.

Umfangreicher noch als AT berichtete die $L W$ über die Wahl. So wurde über die Wahrnehmung und Einschätzungen der Bundestagswahl im Ausland berichtet, und es wurde auch erklärt, wie Wahlprognosen ermittelt werden. Auf die geplante Wahlberichterstattung des Senders am Abend wurde im Laufe des Tages immer wieder hingewiesen. Nach Schließung der Wahllokale stellte die LW u. a. durch kurze Interviews mit Vertretern der Thüringer SPD und der CDU einen Regionalbezug her. Auch auf die Frage der Überhangmandate wurde eingegangen und erläutert, wie diese Mandate zustande kommen.

Im Unterschied zu den anderen Sendern informierte MDR1 zunächst ausführlich über die Wahlergebnisse der Wahl 1998, wobei speziell auf die Ergebnisse in Thüringen eingegangen wurde. Berichtet wurde zudem über Wahlziele und Koalitionsaussagen der im Bundestag vertretenen Parteien bei dieser Wahl und über die letzten Umfrageergebnisse. Am Nachmittag wurden mögliche Ursachen der etwas niedrigeren Wahlbeteili- 
gung erörtert. Zudem wurde live aus zwei Wahllokalen in Thüringen berichtet. Wer am Wahlabend aktuelle und umfangreiche Fakten, ausführliche Live-Berichte und Hintergrundinformationen suchte, der war bei MDR1 am besten aufgehoben. Von 18 bis 23 Uhr wurden bei MDR1 über 70 Beiträge mit einer Gesamtlänge von fast zwei Stunden gesendet. Nach $18 \mathrm{Uhr}$ berichtete MDR1 sehr intensiv über Prognosen, Hochrechnungen und Stellungnahmen der Spitzenpolitiker auf Bundes- und Landesebene. MDR1 ging auch sehr ausführlich auf die Thüringer Ergebnisse ein und reagierte flexibel auf aktuelle Entwicklungen: Als am Wahlabend deutlich wurde, daß die Thüringer Überhangmandate für den Wahlausgang von entscheidender Bedeutung sein könnten, informierte MDR1 detailliert über den Stand der Auszählung der dafür relevanten Erststimmen. Besonders bemerkenswert ist die Tatsache, daß MDR1 bereits am Wahlabend mit der Analyse der Wahlen begann. So wurde ausführlich das Scheitern der PDS kommentiert, und im Unterschied zu allen anderen Sendern gab MDR1 einem Experten, dem Erfurter Kommunikationswissenschaftler Patrick Rössler, mehrmals am Wahlabend die Gelegenheit, seine Einschätzungen des Wahlergebnisses und der Rolle der Medien im Wahlkampf darzustellen.

\subsection{Die Nachwablthematisierung}

Drei Tage später war die Wahl für fünf der sechs Sender nur noch hinsichtlich der Koalitionsverhandlungen von Bedeutung. Die Berichterstattung war weitgehend homogen und offenbar vor allem durch die Zulieferungen der Nachrichtenagenturen geprägt. Unterschiede zwischen den Sendern gab es nur beim Umfang der Berichterstattung und bei der Gewichtung einzelner Aspekte.

Am wenigsten berichtete wiederum $T 40$, aber auch $A T$ hatte kaum mehr wahlbezogene Beiträge. Der Fahrplan für die Koalitionsverhandlungen stand dabei im Mittelpunkt. Nur einmal wurden dabei auch strittige Personalfragen und die Themenfelder genannt, um die es bei den Verhandlungen gehen werde. Die Nachwahlthematisierung von JUMP und $L W$ unterschieden sich nur geringfügig von der bei AT. Nahezu identische Informationen - teilweise sogar die gleichen O-Töne - wurden nach und nach in den Beitrag integriert. Unterschiede kamen bei JUMP dadurch zustande, daß dort auch über die Forderungen berichtet wurde, einen weiteren ostdeutschen Minister ins Kabinett aufzunehmen. Eine bemerkenswerte Besonderheit der LW bestand darin, daß dort der Weiterbau der ICE-Strecke durch den Thüringer Wald thematisiert wurde, ein Projekt, das für Thüringen große Relevanz hat und in den Koalitionsverhandlungen behandelt wurde. Bei MDR1 wurde zwar wesentlich umfangreicher als bei den anderen Sendern über den Beginn der Koalitionsverhandlungen berichtet, aber es wurden im wesentlichen die gleichen Aspekte behandelt. Ausführlicher als bei den anderen Sendern wurde vor allem über die möglichen Streitpunkte in den Verhandlungen thematisiert. Mehr Beachtung fand auch die die ICE-Strecke durch den Thüringer Wald.

Völlig anders stellt sich die Nachwahlthematisierung bei $M K$ dar. Nicht nur zeitlich war sie von allen Sendern am umfangreichsten, sondern sie war auch besonders vielfäl- 
tig und analytisch. Über die Basisinformationen zu den Koalitionsverhandlungen hinaus berichtete der Sender mit O-Tönen über die Ergebnisse der Verhandlungen, insbesondere über die Absicht der Koalition, den Osten stärker zu fördern. Einzig und allein bei MK wurde thematisiert, wie Lobbygruppen versuchen, die Koalitionsverhandlungen zu beeinflussen. Besonders bemerkenswert sind zwei ausführliche, journalistisch anspruchsvolle Expertengespräche (fünf bzw. sieben Minuten) mit Politikwissenschaftlern über die Ursachen und Folgen der Niederlage der PDS und des Erfolges der Grünen.

\section{Zusammenfassung und Schlußfolgerungen}

Alles in allem zeigen die Analysen, daß die Wahl weder im Vorfeld noch in der Nachbereitung bei den hier untersuchten Radiosendern eine bedeutende Rolle gespielt hat. Auch der Wahltag selbst ist bei den meisten Radiosendern ein Tag wie jeder andere.

Hinter diesem generellen Befund verbergen sich jedoch erhebliche Unterschiede zwischen den einzelnen Sendern. In dem für das Publikum attraktivsten Teil des Hörfunkangebots, den AC-Programmen in öffentlich-rechtlicher und privater Trägerschaft (AT, JUMP, LW), taucht das Thema Wahl nur am Rande auf, und dann häufig in einer merkwürdigen Gewichtung von Nachrichtenfaktoren. Aber es herrscht Konsens unter den Verantwortlichen dieser Programme, daß einem politischen Ereignis wie der Bundestagswahl eine gewisse Beachtung geschenkt werden sollte. Die anderen untersuchten Sender weichen in unterschiedlicher Weise von diesem Konsens ab. In einem zweiten Teil des Radiomarktes, hier vertreten durch das jugendorientierte Spartenprogramm TOP40, ist dieser Konsens weitgehend aufgekündigt. Die Wahl spielt dort praktisch keine Rolle. In die andere Richtung weicht das generell stärker informationsorientierte Thüringer Landesprogramm des MDR ab. Dort findet die Wahl - insbesondere am Wahltag - eine größere Beachtung. Das dritte hier untersuchte öffentlich-rechtliche Programm, MDR Kultur, ist zwar auch ein Spartenprogramm, aber nicht nur hinsichtlich der Musik völlig anders als TOP40. MK changiert in der Wahlthematisierung zwischen Mainstream und Informationsorientierung. MDR1 und MK zeigen in ihrer Wahlthematisierung, daß der öffentlich-rechtliche Rundfunk durchaus über journalistisches Potential verfügt, das aber aus unterschiedlichen Gründen - über die man trefflich spekulieren könnte - nur ansatzweise genutzt wird. Problematischer ist jedoch die Tatsache, daß selbst diese publizistische Leistung weiten Teilen des Publikums versagt bleibt, weil durch die Musikfarbe dieser beiden Sender (Schlager bzw. Klassik) große Teile der Hörerschaft von der Nutzung dieser Informationen abgehalten werden. Denn die Musik ist - wie oben bereits dargelegt - das entscheidende Kriterium der Senderauswahl.

Welche Wirkungen diese Art von Wahlthematisierung auf das Publikum hat, darüber kann auf Basis der Inhaltsanalyse nur spekuliert werden. Bei keinem Sender konnte festgestellt werden, da im Rahmen der Wahlthematisierung eine bestimmte Partei oder ein bestimmter Kandidat bevorzugt wurde. Von daher ist eine wahlentscheidende Rolle des Radios nicht sehr wahrscheinlich. Es stellt sich jedoch die Frage, ob das Radio für 
diejenigen ein Ersatz sein kann, die sich von den politischen Informationen in der Zeitung und im Fernsehen bewußt fernhalten. Diese Frage muß sicherlich verneint werden: Wer sich in seiner Wahlinformation nur auf das Radio verläßt, der ist verlassen. Die Zahl der Beiträge, die in irgendeiner Form zur Meinungsbildung in einer speziellen wahlrelevanten Frage hätten beitragen können, war verschwindend gering. Angesichts des schmalen Themenrepertoires mit einem hohen Anteil doch recht abseitiger Themen, der Vermischung unterschiedlicher Genres und der großflächigen Wahlabstinenz, stellt sich statt dessen eher die Frage, welches generelle Politikbild dadurch bei den Radiorezipienten gefördert wird. Sehr unwahrscheinlich ist, daß eine solche Radioberichterstattung die Einflußüberzeugung stärkt und mobilisierend wirkt (vgl. dazu auch die Argumente von Pöttker, 1991, S. 103 und die Befunde von Wolling, 1999, S. 184). Einen gewichtigen Beitrag zur Zivilgesellschaft kann man in der Radiolandschaft nur schwer entdecken.

Welche medienpolitischen Konsequenzen sind aus dem Befund zu ziehen, daß die Bundestagswahl, der Inbegriff politischen Lebens in der Bundesrepublik, bei vielen Radiosendern nur eine sehr eingeschränkte Aufmerksamkeit findet? Wenig sinnvoll und wenig erfolgreich dürfte es sein, Auflagen für den privaten Rundfunk zu verstärken, denn hier ist der Spielraum recht klein bemessen. Mit aller Vorsicht, die unsere relativ kleine empirische Basis gebietet, kann man allerdings die Frage stellen, mit welcher Begründung öffentliche Ressourcen (vor allem Gebühren und Frequenzen) für solche Programme zur Verfügung gestellt werden, die publizistisch nur schwach profiliert sind. Entweder sollte die publizistische Leistung bei denen, die den Auftrag haben und grundsätzlich dazu in der Lage sind, konsequenter eingefordert werden, oder es sollte ein größerer Teil des Hörfunkbereiches marktmäßig organisiert werden. Das würde bedeuten: keine Subventionierung von Anbietern durch Gebühren oder durch kostenlose Überlassung von Frequenzen.

Auch für die weitere Forschung ergeben sich aus der Studie einige Konsequenzen. Eine der größten Herausforderungen für die Inhaltsanalyse von Politik im Radio ist sicherlich die angemessene Abbildung des Beitragstyps, da bei vielen der untersuchten Beiträge eine Einordnung in gängige Genrekategorien schwierig war. Dafür wäre es beispielsweise wichtig, die Ernsthaftigkeit der Berichterstattung in mehreren Abstufungen zu kodieren und dafür entsprechende Indikatoren zu entwickeln. Die erstaunlich große Bedeutung des Humors läßt es geboten erscheinen, die entsprechenden Genres differenzierter zu erfassen, z. B. hinsichtlich der Aggressivität des verwendeten $\mathrm{Hu}$ mors. Notwendig wäre es auch, die Zentralität der behandelten Themen für die Wahl und den Wahlkampf zu erheben, um einen Eindruck zu bekommen, inwieweit der Versuch unternommen wurde, mit der Wahlthematisierung zur demokratischen Willensbildung beizutragen. Eine besondere Herausforderung stellt die prozessuale Dimension des Hörfunkprogramms dar. Es gilt ein geeignetes Instrument zu entwickeln, um die Dynamik von Themen zu erfassen. Die untersuchten Sender unterschieden sich z. T. deutlich dahingehend, ob und in welchem Maße sie die Berichterstattung zu einzelnen Wahlkampfthemen im Laufe des jeweiligen Tages ergänzten und weiterentwik- 
kelten. Außerdem wäre eine methodische Konvention zu begrüßen, wie mit den Wiederholungen von Beiträge umzugehen ist; ob und in welcher Weise dies bei Inhaltsanalysen von Radioprogrammen berücksichtigt werden sollte. Auch die unterschiedliche Chance der Wahrnehmung von Programmen im Tagesablauf müßte bei der Analyse verstärkt berücksichtigt werden. Von großer Bedeutung wäre darüber hinaus die Codierung der Urheberschaft des Wahlbezugs: Durch wen wurde der Wahlbezug in den Beiträgen hergestellt und welcher Eindruck wird damit erweckt? Der Bezug zur Wahl wird nämlich nicht nur dazu verwendet, die Wichtigkeit eines Beitrags oder eines Themas hervorzuheben, sondern im Gegenteil: Ein Thema in den Zusammenhang mit der Wahl zu bringen, ist von seiten der Radiojournalisten eine Form der Reaktanz. Es ist oft der Versuch, etwas als versteckten Wahlkampf zu "entlarven". Wahlbezug heißt somit häufig: Das ist nicht ernst zu nehmen, es ist ja nur Wahlkampf? Und das ist durchaus ernst zu nehmen.

\section{Literatur}

Arnheim, R. (1936). Radio. London: Faber \& Faber.

Becker, L. B., \& Dunwoody, S. (1982). Media use, public affairs knowledge and voting in a local election. Journalism Quarterly, 59, 212-218, 255.

Berelson, B. R., Lazarsfeld, P. F., \& McPhee, W. N. (1954). Voting. A study of opinion formation in a presidential campaign. Chicago, IL: University of Chicago Press.

Berens, H., Kiefer, M.-L., \& Meder, A. (1997). Spezialisierung der Mediennutzung im dualen Rundfunksystem. Media Perspektiven, o. Jg.(2), 80-91.

Cantril, H. (1947). The invasion from Mars: A study in the psychology of panic. Princeton, NJ: Princeton University Press.

Dahlem, S. (2001). Wablentscheidung in der Mediengesellschaft. Freiburg. Alber.

Denton, R. E. (Hrsg.). (1998). The 1996 presidential campaign. A communication perspective. Westport, CT.: Praeger.

Ecke, J.-O., \& Stuiber, H.-W. (1995). Nachrichten im Hörfunk. Hinweise auf ihre Bedeutung und Bewertung. In H.-J. Bucher, W. Klingler \& C. Schröter (Hrsg.), Radiotrends. Formate, Konzepte und Analysen (S. 163-178). Baden-Baden: Nomos.

Gleich, U. (1995). Hörfunkforschung in der Bundesrepublik. Methodischer Überblick, Defizite und Perspektiven. Media Perspektiven, o. J.(11), 554-561.

Gleich, U. (2000). Nutzungsmotive und Funktionen des Radios. Media Perspektiven, o. Jg.(9), 427-432.

Hasebrink, U. (1994). Hörfunk - ein politisches Informationsmedium? Ein Überblick über Forschungsergebnisse zur Hörfunknutzung. In O. Jarren (Hrsg.), Politische Kommunikation im Hörfunk und Fernsehen. (S. 157-172). Opladen: Leske + Budrich.

Hesse, K. R. (1994). Das politische Informationsangebot im Hörfunk: Ergebnisse von ProgrammInhaltsanalysen. In O. Jarren (Hrsg.), Politische Kommunikation im Hörfunk und Fermsehen. (S. 149-156). Opladen: I eske + Budrich.

Hollander, B. A. (1996). 'Talk Radio: Predictors of use and effects on attitudes about government. Journalism Quarlerly, 73, 102-113. 
Holtz-Bacha, C. (1997). Hörfunk-Wahlkampagnen: einflußreich - auch einfallsreich? Zeitschrift für Parlamentsfragen, 28, 294-310.

Holtz-Bacha, C. (Hrsg.). (1999). Wablkampf in den Medien - Wablkampf mit den Medien. Ein Reader zum Wabljabr 1998. Opladen: Westdeutscher Verlag.

Holtz-Bacha, C., \& Kaid, L. L. (Hrsg.). (1996). Wablen und Wablkampf in den Medien. Untersuchungen aus dem Wahljabr 1994. Opladen: Westdeutscher Verlag.

Jarren, O., \& Bode, M. (1996). Ereignis- und Medienmanagement politischer Parteien. Kommunikationsstrategien im superwahljahr 1994. In Bertelsmann Stiftung (Hrsg.), Politik überzeugend vermitteln. Wablkampfstrategien in Deutschland und den US A (S. 65-114). Gütersloh: Verlag Bertelsmann-Stiftung.

Jarren, O., \& Donges, P. (1996). Keine Zeit für Politik? Landespolitische Berichterstaltung im Rundfunk: Joumalisten, Offentlichleeitsarbeiter und Politiker in der Interaktion; das Beispiel Hamburg. Berlin: Vistas.

Kiefer, M.-L. (1996). Massenkommunikation V. Eine Langzeitstudie zur Mediennutzung und Medienbewertung 19641995. Baden-Baden: Nomos.

Kindel, A. (1998). Erinnern von Radio-Nachrichten. Eine empirische Studie über die Selektionsleistungen der Hörer von Radio-Nachrichten. München: Reinhard Fischer.

Klingler, W., \& Schröter, C. (1993). Strukturanalysen von Radioprogrammen 1985 bis 1990 . Eine Zwischenbilanz der Hörfunkforschung im dualen Rundfunk. Media Perspektiven, o. Jg.(10), 479-490.

Klingler, W., \& Windgasse, T. (1994). Der Stellenwert des Hörfunks als Informationsquelle in den 90er Jahren. In M. Jäckel \& Winterhoff-Spurk (Hrsg.), Politische Kommunikation in Hörfunk und Fernsehen. (S. 103-122). Berlin: Vistas.

Krüger, U. M., \& Zapf-Schramm, T. (2002). Wahlberichterstattung im öffentlich-rechtlichen und privaten Fernsehen. Media Perspektiven, o. Jg. (12), 610-622.

Lazarsfeld, P. F., Berelson, B., \& Gaudet, H. (1944). The people's ihoice. How the voter makes up bis mind in a presidential campaign. New York: Duell, Sloan and Pearce.

Lohmann, M. (1987). Hörfunk mit Schlagseite: O-'Ton für die Regierung. Radioszene Norddeutschland: Vier Sender zur Auswahl. Neue Medien, (11), S. 71-73.

Marcinkowski, F. (1998). Politikvermittlung durch Fernsehen und Hörfunk. In U. Sarcinelli (Hrsg.), Politik. termiltlung und Demokratie in der Mediengesellschaft (S. 165-183). Opladen: Westdeutscher Verlag.

Merton, R. K. (1946). Mass persuasion: The social psychology of a war bond drive. New York: Harper \& Brothers.

Meyer, B., \& Port, J. (1994). Politik und Kommunikation / Politik in den Medien. Schwerpunkt: Hörfunk und Fernsehen (Inhalte, Rezeption, Wirkung); eine Auswahlbibliographie. In O. Jarren (Hrsg.), Politische Kommunikation im Hörfunk und Fernseben (S. 209-233). Opladen: Leske + Budrich.

Müller, M. G. (1999). Parteienwerbung im Bundestagswahlkampf 1998. Media Perspektiven, o. Jg.(5), 251-261.

Oehmichen, E., \& Ridder, C.-M. (Hrsg.). (2003). Die MedienNutzerTypologie. Ein newer Ansatz der Publikumsanalyse. Baden-Baden: Nomos.

Oehmichen, E. (1999). Die MedienNutzer'lypologie als Beratungsinstrument im Hörfunk. Zur Umsetzung der Publikumstypologie von ARD und 'ZDF für Planungsprozesse. Media Perspektiven, o. Jg.(10), 549-556.

Ohr, D., \& Schrott, P. R. (2001). Campaigns and information seeking. Evidence from a German state election. European Journal of (ommunication, 16, 419-449). 
Pöttker, H. (1991). Dualer Rundfunk und Politikverdrossenheit. Zur fortschreitenden Ausdifferenzierung von Öffentlichkeit in modernen Gesellschaften. In S. Müller-Doohm \& K. Neumann-Braun (Hrsg.), Offentlichkeit Kultur Massentommunikation (S. 91-109). Oldenburg. BIS-Verlag

Rettich, M., \& Schatz, R. (1998). Amerikanisierung oder Die Macht der Themen. Bonn: Innovatio.

Ridder, C.-M., \& Engel, B. (2001). Massenkommunikation 2000: Images und Funktionen der Massenmedien im Vergleich. Ergebnisse der 8. Welle der ARD/ZDF-Langzeitstudie zur Mediennutzung und -bewertung. Media Perspektiven, o. Jg.(3), S. 102-125.

Scherer, H. (1995). Kommunikationskanäle in der Europawahl 1989. Eine international vergleichende Studie. In L. Erbring (Hrsg.), Kommunitationsraum Europa (S. 203-221). Konstanz: Ölschläger.

Schönbach, K. (1983). Das unterschätzte Medium. Politische Wirkungen von Presse und Fernseben im Vergleich. München: Saur.

Schönbach, K., \& Goertz, L. (1995). Radio-Nacbrichten: Bunt und flücbtig? Eine Untersuchung zu Präsentationsformen von Hörfunknachrichten und ibren Leistungen. Berlin: Vistas.

Schulz, W. (1998). Media change and the political effects of television: Americanization of the political culture? Communications, 23, 527-543.

Schulz, W. (2001). Politische Mobilisierung durch Mediennutzung? Beziehungen zwischen Kommunikationsverhalten, politischer Kompetenz und Partizipationsbereitschaft. In A. Koch, M. Wasmer \& P. Schmidt (Hrsg.), Politische Partizipation in der Bundesrepublik Deutschland. Empirische Befunde und theoretische Erklärungen. (S. 169-194). Opladen: Westdeutscher Verlag.

Schulz, W., Zeh, R., \& Quiring, O. (2000). Wählerverhalten in der Mediendemokratie. In M. Klein, W. Jagodzinski, E. Mochmann \& D. Ohr (Hrsg.), 50 Jahre Empirische Wablforschung (S. 413-443). Opladen: Westdeutscher Verlag.

Semetko, H. A., \& Schönbach, K. (1994). Ciermanys "Unity Election": Voters and the media. Cresskill, NJ.: Hampton Press.

Shapiro, M. A., \& Rieger, R. H. (1992). Comparing positive and negative political advertising on radio. Joumalism Quarterly, 69, 135-145.

Trebbe, J., \& Maurer, T. (1999). Hörfunklandschaft Niedersachsen 1998: Eine vergleichende Analyse der öffentlichrechtlichen und privaten Radiosender. Berlin: Vistas.

Weiß, R. (1988). Programmstrukturen im dualen Hörfunksystem: Engebnisbericht über die inhaltsanalytische Untersuchung der Programmstruktur des Hörfunkangebotes in Hamburg. Hamburg. Hans-Bredow-Institut.

Widlok, P. (1994). Hörfunkanbieter und Hörfunkprogramme in Deutschland. In O. Jarren (Hrsg.), Politische Kommunikation in Hörfunk und Fernseben. (S. 135-148). Opladen: Leske+Budrich.

Wolling, J. (1999). Politikverdrossenheit durch Massenmedien? Der Einfluss der Medien auf die Einstellungen der Bürger zur Politik. Opladen: Westdeutscher Verlag. 